\title{
Accuracy of machine learning for differentiation between optic neuropathies and pseudopapilledema
}

\author{
Jin Mo Ahn', Sangsoo Kim, Kwang-Sung Ahn², Sung-Hoon Cho² and Ungsoo S. Kim ${ }^{3^{*}}$ (D)
}

\begin{abstract}
Background: This study is to evaluate the accuracy of machine learning for differentiation between optic neuropathies, pseudopapilledema (PPE) and normals.

Methods: Two hundred and ninety-five images of optic neuropathies, 295 images of PPE, and 779 control images were used. Pseudopapilledema was defined as follows: cases with elevated optic nerve head and blurred disc margin, with normal visual acuity (>0.8 Snellen visual acuity), visual field, color vision, and pupillary reflex. The optic neuropathy group included cases of ischemic optic neuropathy (177), optic neuritis (48), diabetic optic neuropathy (17), papilledema (22), and retinal disorders (31). We compared four machine learning classifiers (our model, GoogleNet Inception v3, 19-layer Very Deep Convolution Network from Visual Geometry group (VGG), and 50-layer Deep Residual Learning (ResNet)). Accuracy and area under receiver operating characteristic curve (AUROC) were analyzed.
\end{abstract}

Results: The accuracy of machine learning classifiers ranged from 95.89 to $98.63 \%$ (our model: $95.89 \%$, Inception V3: 96.45\%, ResNet: 98.63\%, and VGG: 96.80\%). A high AUROC score was noted in both ResNet and VGG (0.999).

Conclusions: Machine learning techniques can be combined with fundus photography as an effective approach to distinguish between PPE and elevated optic disc associated with optic neuropathies.

Keywords: Machine learning, Pseudopapilledema, Optic neuropathy, Optic disc swelling

\section{Background}

Pseudopapilledema (PPE) is defined as an optic nerve with an elevated optic disc and blurred margins that is similar to papilledema or disc swelling associated with various optic neuropathies [1]. Although PPE is a benign condition, it should be differentiated from other optic neuropathies to reduce the need for unnecessary examination and to provide precise diagnosis, prognosis and therapeutic options to the patients. Recently, multimodal imaging analysis including B-scan ultrasonography, fundus photography, autofluorescence, fluorescein angiography, and optical coherence tomography (OCT) have provided useful information for exact diagnosis of

\footnotetext{
* Correspondence: ungsookim@kimeye.com

${ }^{3}$ Department of Ophthalmology, Kim's Eye Hospital, Youngshin-ro 136,

Youngdeungpo-gu, Seoul 150-034, South Korea

Full list of author information is available at the end of the article
}

PPE [2-4]. However, the exact differentiation is still difficult.

Machine learning is the use of artificial computer intelligence to enable computers to learn automatically, without being programmed. In ophthalmology, machine learning has been used to analyze various disorders such diabetic retinopathy age-related macular degeneration, and glaucoma [5-8]. We investigated the accuracy and sensitivity of machine learning for differentiation between PPE, optic neuropathies and normals.

\section{Methods \\ Patients}

Pseudopapilledema was defined as follows: cases with an elevated optic nerve head and blurred disc margins, with normal visual acuity ( $>0.8$ Snellen visual acuity), visual field, color vision, and pupillary reflex. Only those patients who did not change their optic nerve head and 
visual function for more than one year were included in the present study. The optic neuropathies group includes 177 cases of ischemic optic neuropathy, 48 of optic neuritis, 17 of diabetic optic neuropathy, 22 of papilledema, and 31 of retinal disorders such as central retinal vein occlusion or posterior uveitis (Fig. 1a). Normal controls were enrolled from routine examination without any abnormal findings and visual problems.

\section{Data preparation}

Fundus photographs were collected from Kim's Eye Hospital. Fundus photography was obtained using a nonmydriatic auto fundus camera (AFC-330, Nidek, Japan). A total of 1369 images were obtained, including 295 images of optic neuropathies, 295 of PPE, and 779 normal control images. The obtained images were scaled to a fixed width of 500 pixels while keeping the aspect ratio constant. To remove variations in lightning and brightness of images, the local average color was subtracted using Gaussian filtering [9]. Finally, pixels of each image was normalized to have 0 mean and 1 standard deviation. In order to produce fixed-size input necessary for machine learning models, each photos were cropped with size of $240 \times 240$ pixels at the region of optic nerve. Figure $1 \mathrm{~b}$ shows the schematic view of the image preprocessing step. The entire set of 1369 images were split into an 876-image training dataset for training the model, a 274-image validation dataset for validation of a

b
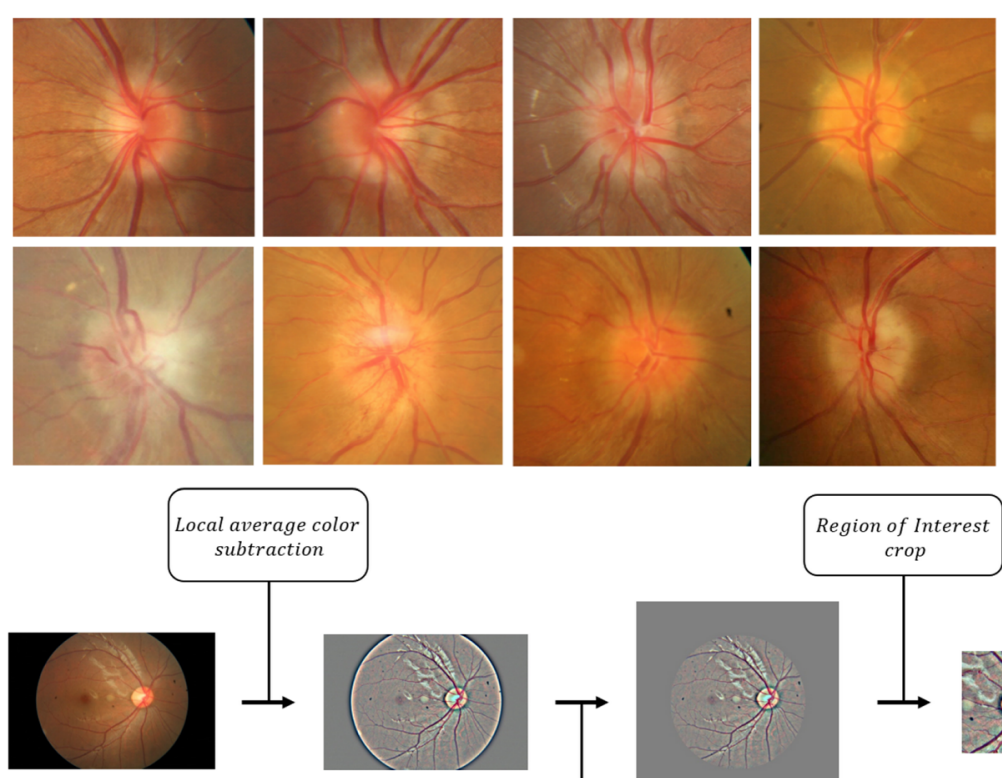
crop
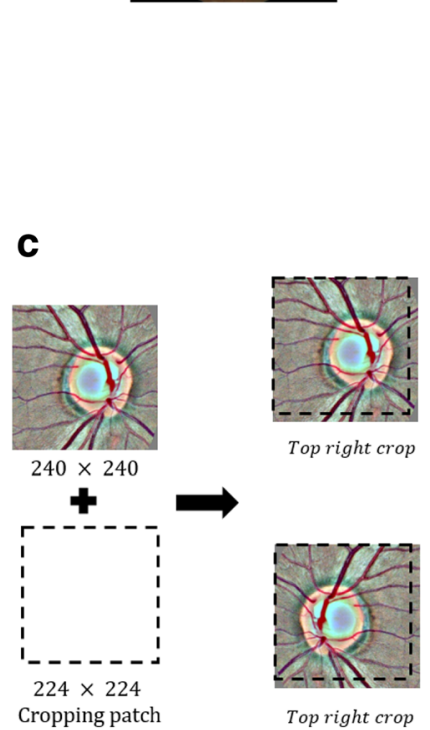

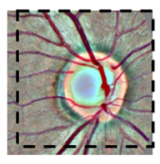

r-t-

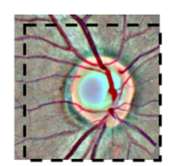

Bottom left crop

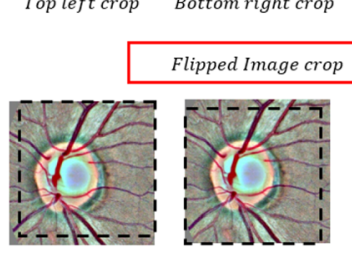

Top left crop

Bottom right crop

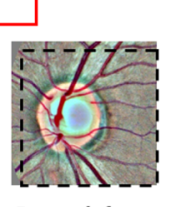

Bottom left crop

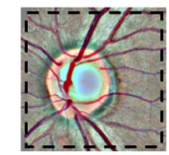

Top right crop

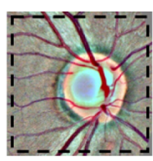

Center crop

Center crop

Fig. 1 a Optic disc findings in fundus photography. Various features from pseudopapilledema (upper low) and swollen disc from optic neuropathies (lower low). b Schematic view of image pre-processing process c Schematic view of image augmentation process 
the model while training, and a 219-image test dataset for evaluation of the final model. The validation dataset was generated by a random split of $20 \%$ of the entire dataset; the test dataset was generated by a random split of $20 \%$ of the remaining images after validation split (Table 1). Normal and PPE patients had normal findings on OCT (Cirrus HD-OCT, Carl Zeiss Meditec Inc., Dublin, CA and Spectralis, Heidelberg Engineering, Heidelberg, Germany), and visual field tests (Humphrey 740 visual field analyzer, Carl Zeiss Meditec Inc., Dublin, CA).

\section{Convolutional neural network Data augmentation}

To overcome possibility of overfitting the model due to small dataset, image augmentation technique was applied to each image. Augmentation process was conducted by cropping all four corners of an image and in the middle generating five images with a fixed size of $224 \times 224$ pixels. This cropping process was repeated after flipping the image which creates 10 augmented images from a single original image. Providing augmented images to a machine learning model can help overcome overfitting and to better in decision-making due to enlarged dataset with different pixel representation [10]. Figure 1c shows the shows augmentation process.

\section{Training model}

We have constructed a convolutional neural network, using Google's Tensorflow deep learning framework as backend [11]. In order to produce best working model, an optimum set of working hyper-parameters are needed. These hyper-parameters include learning rate, activation function, patch size, filter size, number of fully connected layers, and number of hidden nodes in each fully connected layer. However, trying out all possible combinations of hyper-parameters is very time consuming and computationally expensive. Many methods have been proposed for hyper-parameter tuning such as grid search, random search [12], genetic algorithm [13], and Bayesian optimization [14]. We implemented Bayesian optimization for our hyper-parameter tuning process using python package Scikit-Optimize. Seven hyper-parameters were tuned using Bayesian optimization including number of convolution layers, number of convolution filters, number of convolution patch size,

Table 1 Sample number for Convolutional Neural Network

\begin{tabular}{lllll}
\hline & Normal & Pseudopapilledema & Papilledema & Total \\
\hline Entire Data & 779 & 295 & 295 & 1369 \\
Training Data & 505 & 197 & 174 & 876 \\
Validation Data & 155 & 53 & 66 & 274 \\
Test Data & 119 & 45 & 55 & 219 \\
\hline
\end{tabular}

number of fully connected layers, number of hidden nodes in each fully connected layer, activation function (rectifier linear unit, exponential linear units, hyperbolic tangent), and learning rate. Max pooling layers were fixed after every convolutional layer with patch size $2 \times 2$ and stride 2 , and dropout layers with rate 0.5 were fixed after every fully connected layer. Mattern kernel was used for Bayesian optimization and expected improvement was used for acquisition function. The best hyperparameters were selected after 100 rounds of updating the Gaussian process model. Figure 2 shows a schematic view of hyper-parameter tuning process. The training was conducted again with the selected hyper-parameters with Adam optimizer [15] and cross entropy as a loss function until the average loss of validation data for each epoch started to increase.

\section{Transfer learning}

We conducted transfer learning [16], which involved training with a predefined (trained) model using three well-known convolutional neural networks. These include GoogleNet Inception v3 [17], 19-layer Very Deep Convolution Network from Visual Geometry group (VGG) and 50-layer Deep Residual Learning also known as ResNet [18, 19]. These networks were trained using approximately 1.2 million images from ImageNet LargeScale Visual Recognition Challenge. We modified the fully connected layers of the three networks to fit our classification needs. Bayesian optimization was used to tune the hyper-parameters. Four hyper-parameters were tuned including number of fully connected layers, number of hidden nodes, activation function, and learning rate. Dropout layers with rate 0.5 were fixed after every fully connected layer. Fine-tuning was conducted after hyper-parameter tuning using Adam optimizer and cross entropy as a loss function. Training was considered finished when the average loss of validation data for each epoch started to increase.

\section{Evaluation}

The model obtains an image and conduct mathematical calculation defined by training the model and ultimately outputs three probabilities. These probabilities represent a probability of being photograph of a normal subject, PPE and papilledema. Since we used augmented data (10 images per photography), we generated 30 probabilities (10 for each class) from a single image. By averaging these probability values by class, we obtained three probabilities which corresponds to image being normal, PPE and papilledema (Fig. 3b). The model makes decision by choosing the class with maximum probability. Using this strategy, we evaluated our model as well as GoogleNet Inception v3, VGG, and ResNet transferred model. Also, we have calculated 


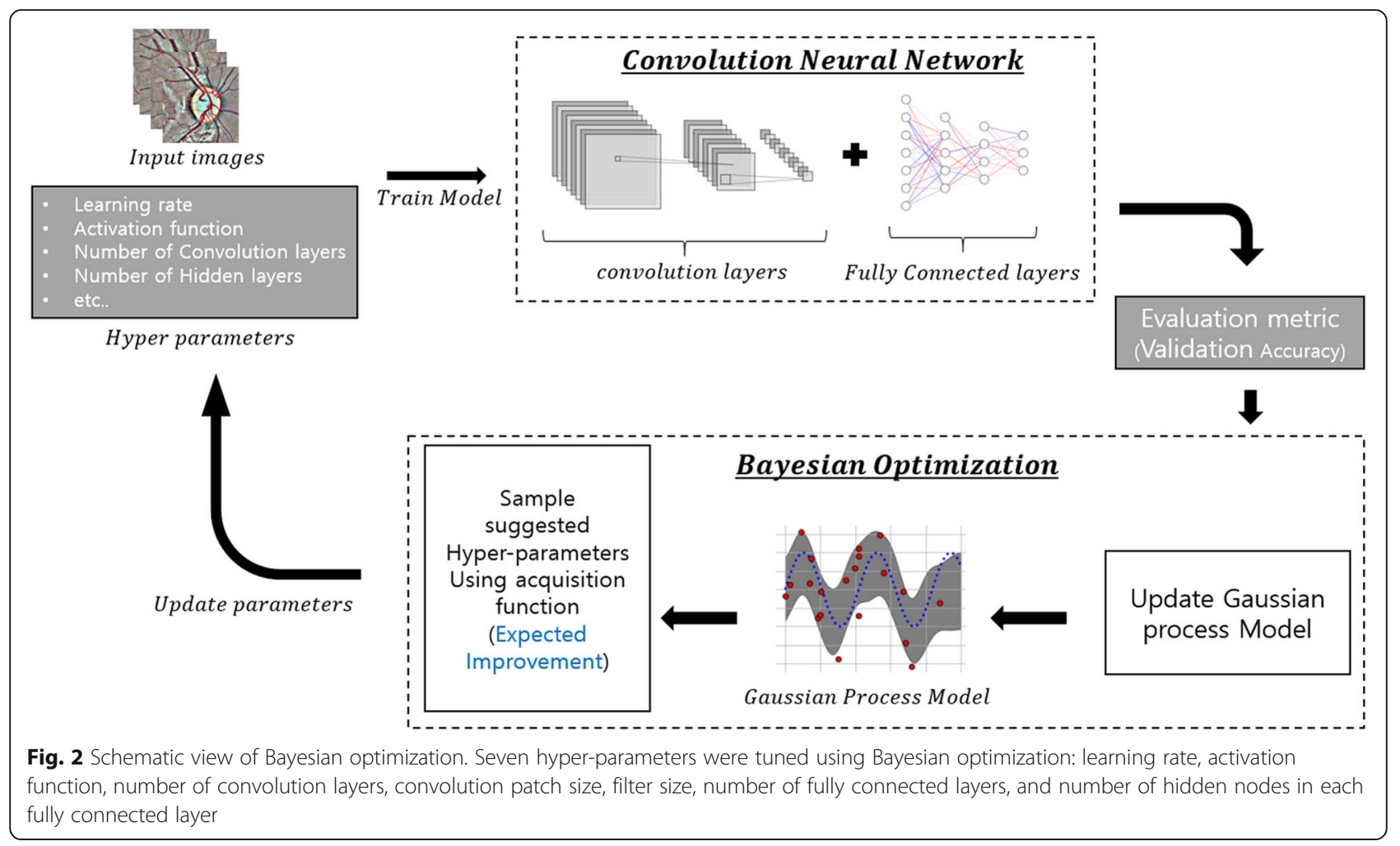

micro-averaged sensitivity and specificity of each model and generated ROC (receiver operating characteristic) curve which indicates overall performance of how well the models classify images into three groups (Normal, PPE, papilledema).

\section{Results}

Table 2 shows the summarized results of our model and transfer learning model. After hyper-parameter tuning, our model exhibited 3 convolution layers and 5 fully connected layers. The first convolution layer had a patch size

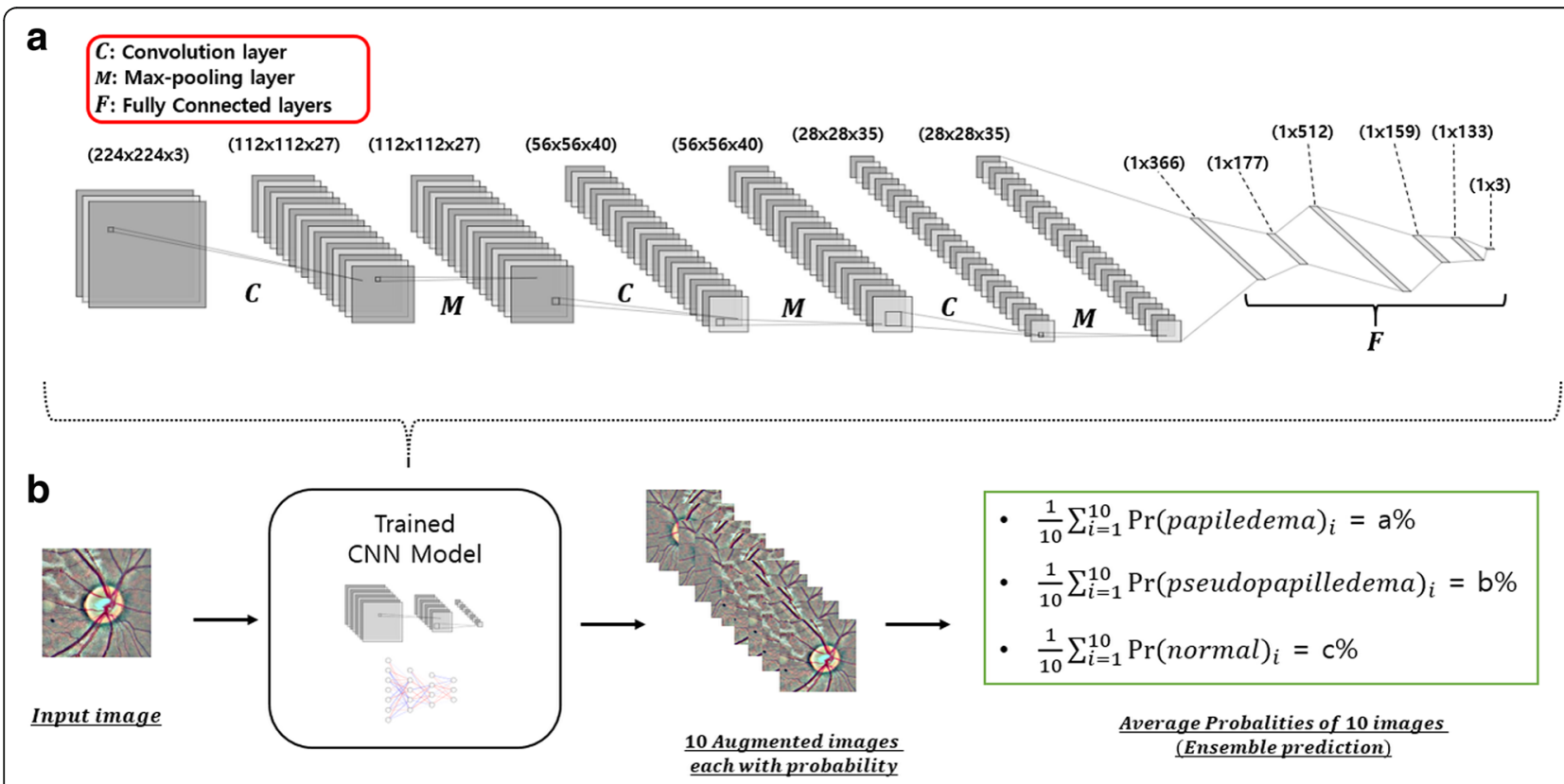

Fig. 3 a. Schematic view of our model. It consists of 3 convolutional layer each with max pooling layer followed by 5 fully connected layers and a softmax layer. b. Evaluation process. Ten augmented images were averaged to give a single probability for each class 
Table 2 Evaluation of our model and transferred model

\begin{tabular}{|c|c|c|c|c|c|c|c|c|}
\hline & \multicolumn{2}{|l|}{ Our Model } & \multicolumn{2}{|l|}{ Inception V3 } & \multicolumn{2}{|l|}{ ResNet } & \multicolumn{2}{|l|}{ VGG } \\
\hline & Ensemble Accuracy & $\overline{A U R O C}$ & Ensemble Accuracy & $\overline{A U R O C}$ & Ensemble Accuracy & $\overline{\text { AUROC }}$ & Ensemble Accuracy & $\overline{A U R O C}$ \\
\hline Training Data & $100 \%$ & 1.0 & $100 \%$ & 1.0 & $100 \%$ & 1.0 & $100 \%$ & 1.0 \\
\hline Validation Data & $96.35 \%$ & 0.989 & $98.18 \%$ & 0.993 & $98.18 \%$ & 0.996 & $97.81 \%$ & 0.996 \\
\hline Test Data & $95.89 \%$ & 0.992 & $96.35 \%$ & 0.997 & $98.63 \%$ & 0.999 & $96.80 \%$ & 0.999 \\
\hline
\end{tabular}

of $2 \times 2$ with 27 filters, the second had a patch size of $12 \times$ 12 with 40 filters, and the last convolution layer had a patch size of $29 \times 29$ with 35 filters. Max pooling layer was applied after every convolutional layer with a patch size of $2 \times 2$ and a stride of 2 . The fully connected layers consisted of 366, 177, 512, 159, and 133 hidden nodes, respectively. A dropout rate of 0.5 was used in fully connected layers. As for activation function, ReLu (Rectifier linear unit) was used.

Figure 3a shows the schematic architecture of our CNN model. The Inception V3 model exhibited 1 fully connected layer with 60 hidden nodes along with Rectifier linear unit as an activation function. The VGG model exhibited 3 fully connected layers with each layer having 512 hidden nodes and Exponential linear unit as an activation function. The ResNet model had 1 fully connected layer with 325 hidden nodes with hyperbolic tangent as an activation function. All the transferred models had dropout layer with dropout rate 0.5 after every fully connected layer. Further, all models used softmax layer as a classification layer. The best performing model based on test accuracy was the ResNet transfer learned model. The ROC curve for each model is depicted in Fig. 4a and the confusion matrix based on test data for each model is depicted in Fig. 5. At the cost of 0.007 difference of AUROC, our model used the least number of parameters $(11,636,096)$ among models (Fig. 4-b). In addition, the validation loss graph showed that validation loss reached zero level at around epoch 16 (Fig. 4c).

\section{Discussion}

This study suggests that machine learning techniques can be combined with fundus photography as an effective approach to distinguish between PPE and elevated optic disc related with optic neuropathies.

We have used 3 state-of-the-art convolutional neural networks, including GoogleNet Inception V3, VGG, and ResNet. In addition, we used pre-trained weights from ImageNet Large-Scale Visual Recognition Challenge as the initial parameter to train our model instead of random weights; this is a popular method since these initial parameters are already optimized for detecting natural images such as edges and curves [20], thus solving the issue of overfitting when not many data are available. We have also trained our own model from scratch using Bayesian optimization as the hyper-parameter tuning process. As depicted in Table 2, transferred models outperformed our model based on test accuracy but, the difference, based on AUROC, was small. Between our model and the best performing ResNet transferred model, our model used far less parameters, which is computationally effective.

Overfitting, which refers to models performing well on the trained data but not well on unseen data is a common issue when a small dataset is used to train the model [21]. Since our dataset consisted of only $1369 \mathrm{im}$ ages, there might have been a possibility of overfitting. However, we addressed this issue by incorporating regularization techniques such as adding dropout layers and data augmentation. Dropout randomly corrupts hidden nodes between layers which changes the detail of the model every training iteration [22]. Thus, this process leads to a more generalized model when a sufficient number of training iterations are given. Data augmentation allows the machine to learn an image from different views. This technique can also help overcome
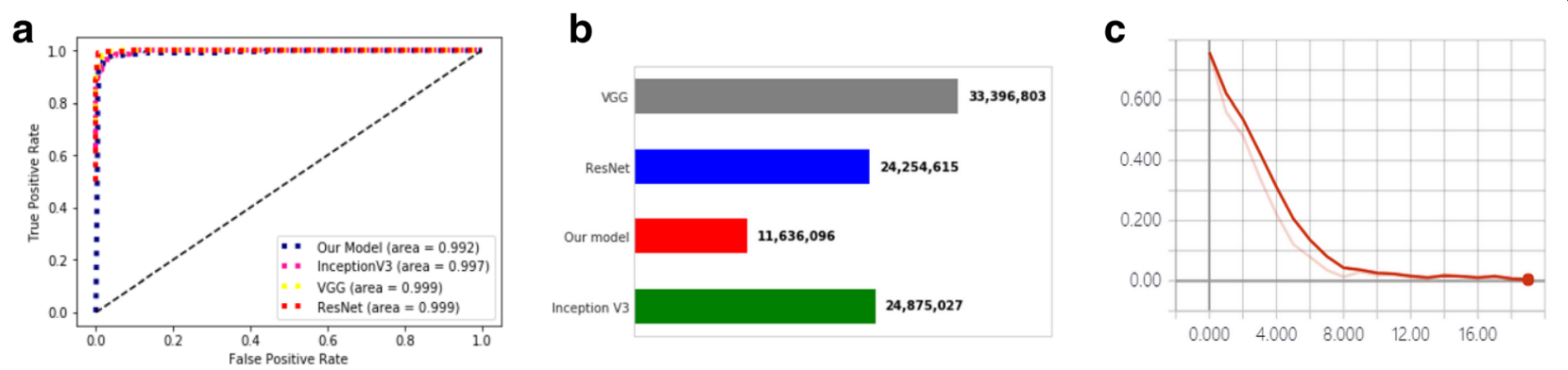

Fig. 4 a. Receiver operating characteristic curve. b. Number of parameter comparison between models. c. Loss graph for our model. Y-axis indicates loss for validation data and X-axis indicates number of epoch 


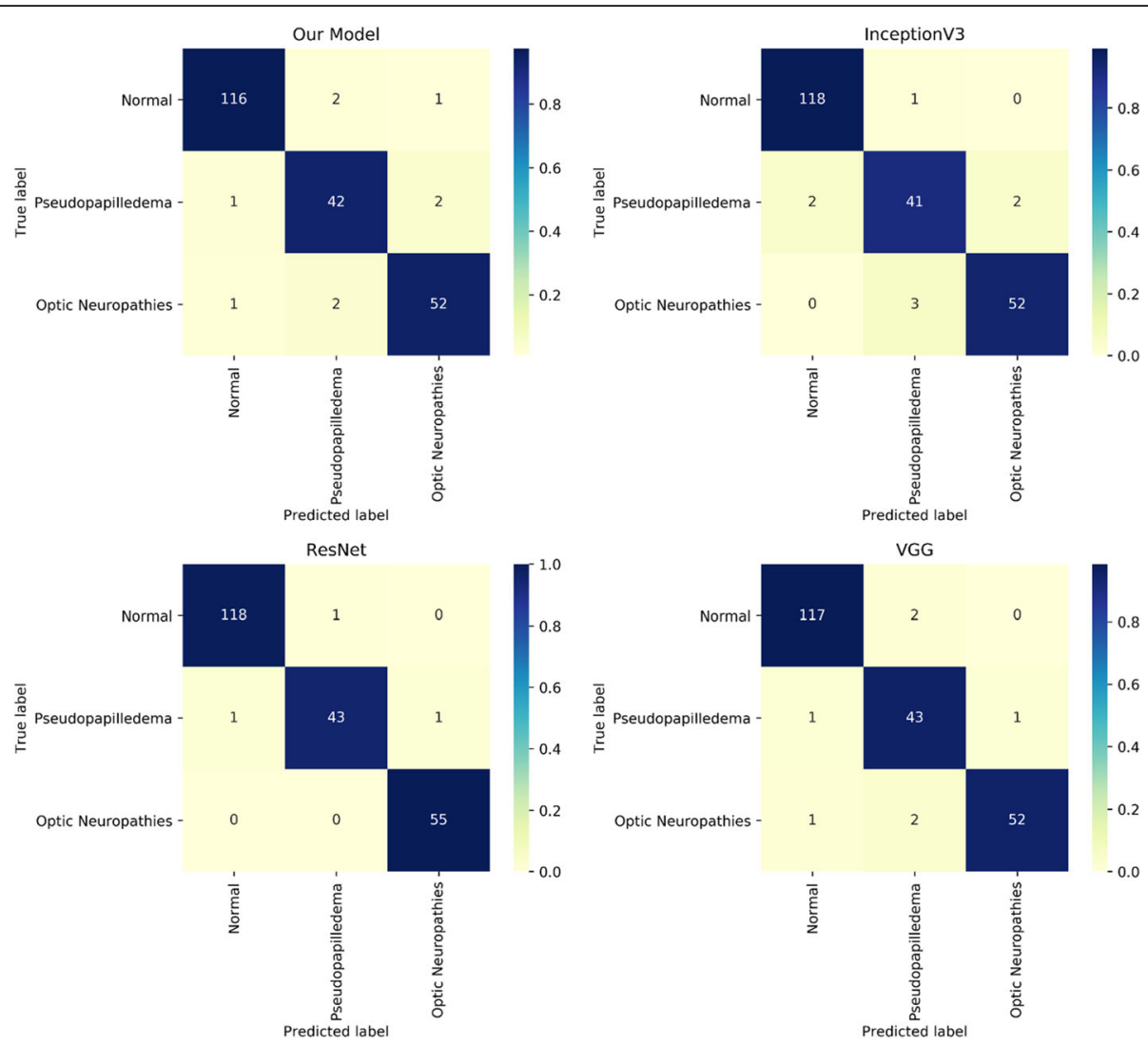

Fig. 5 Confusion matrix

the issue of small training dataset by generating many augmented images. The validation loss graph for our model indicates that our model reached minimum, which is an indication of an optimal model [10].

Even though we have generated our own model and used well-known, state-of-the-art convolutional neural networks, getting an insight into how a machine classifies a fundus photo as normal or disease status can be a challenging task. Therefore, further study is needed into visualizing the convolution layers and filters to get an idea of how machines classify images. Further, larger scale datasets may help validate our findings.

\section{Conclusions}

Machine learning techniques can be combined with fundus photography as an effective approach to distinguish between PPE and elevated optic disc associated with optic neuropathies.

\section{Abbreviations}

OCT: Optical coherence tomography; PPE: Pseudopapilledema; VGG: Visual Geometry group

\section{Acknowledgements}

None.

\section{Authors' contributions}

All authors participated in design of the study. JMA, SHC and USK analyzed and interpreted the data. JMA and USK were major contributors in writing manuscript. KSA, SSK and USK supervised the manuscript. All authors read and approved the final manuscript.

Funding

None.

Availability of data and materials

The datasets analyzed during the current study are available from the corresponding author (ungsookim@kimeye.com) on reasonable request.

Ethics approval and consent to participate

This retrospective study was approved by the institutional review board of Kim's Eye Hospital which waived the requirement for informed consent from participants. It was conducted in accordance with all relevant requirements of the Declaration of Helsinki.

Consent for publication

Not applicable.

\section{Competing interests}

The authors declare that they have no competing interests.

\section{Author details}

'Department of Bioinformatics and Life Science, Soongsil University, Seoul, South Korea. ${ }^{2}$ Functional Genome Institute, PDXen Biosystems Inc, Seoul, Republic of Korea. ${ }^{3}$ Department of Ophthalmology, Kim's Eye Hospital, Youngshin-ro 136, Youngdeungpo-gu, Seoul 150-034, South Korea. 
Received: 4 January 2019 Accepted: 29 July 2019

Published online: 09 August 2019

\section{References}

1. Trick GL, Bhatt SS, Dahl D, Skarf B. Optic disc topography in pseudopapilledema: a comparison to pseudotumor cerebri. J Neuroophthalmol. 2001;21:240-4.

2. Aghsaei Fard M, Okhravi S, Moghimi S, Subramanian PS. Optic nerve head and macular optical coherence tomography measurements in papilledema compared with Pseudopapilledema. J Neuroophthalmol. 2018.

3. Thompson AC, Bhatti MT, El-Dairi MA. Bruch's membrane opening on optical coherence tomography in pediatric papilledema and pseudopapilledema. J AAPOS. 2018;22:38-43 e3.

4. Saenz R, Cheng H, Prager TC, Frishman L, Tang RA. Use of A-scan ultrasound and optical coherence tomography to differentiate papilledema from Pseudopapilledema. Optom Vis Sci. 2017;94:1081-9.

5. Gulshan V, Peng L, Coram M, et al. Development and validation of a deep learning algorithm for detection of diabetic retinopathy in retinal fundus photographs. JAMA. 2016;316:2402-10.

6. Kim SJ, Cho KJ, Oh S. Development of machine learning models for diagnosis of glaucoma. PLoS One. 2017;12:e0177726.

7. Rahimy E. Deep learning applications in ophthalmology. Curr Opin Ophthalmol. 2018;29:254-60.

8. Ahn JM, Kim S, Ahn KS, Cho SH, Lee KB, Kim US. A deep learning model for the detection of both advanced and early glaucoma using fundus photography. PLoS One. 2018;13:e0207982.

9. Ebner M. Color constancy based on local space average color. Mach Vis Appl. 2009;20:283-301.

10. Wong SC, Gatt A, Stamatescu V, McDonnell MD. Understanding data augmentation for classification: when to warp? arXiv preprint arXiv. 2016: 160908764.

11. Girija SS. Tensorflow: large-scale machine learning on heterogeneous distributed systems. 2016.

12. Bergstra J, Bengio Y. Random search for hyper-parameter optimization. J Mach Learn Res. 2012;13:281-305.

13. Man K-F, Tang K-S, Kwong S. Genetic algorithms: concepts and applications [in engineering design]. IEEE Trans Ind Electron. 1996:43:519-34.

14. Snoek J, Larochelle H, Adams RP. Practical bayesian optimization of machine learning algorithms. Adv Neural Inf Proces Syst. 2012:2951-9.

15. Kinga D, Adam JB. A method for stochastic optimization. International conference on learning representations (ICLR), 2015.

16. George D, Shen H, Huerta E. Deep transfer learning: a new deep learning glitch classification method for advanced LIGO. arXiv preprint arXiv. 2017: 170607446.

17. Szegedy C, Vanhoucke V, loffe S, Shlens J, Wojna Z. Rethinking the inception architecture for computer vision. Proc IEEE Conf Comput Vis Pattern Recognit. 2016:2818-26.

18. Conneau A, Schwenk H, Barrault L, Lecun Y. Very deep convolutional networks for text classification. arXiv preprint arXiv. 2016:160601781.

19. Wu S, Zhong S, Liu Y. Deep residual learning for image steganalysis. Multimed Tools Appl. 2018;77:10437-53.

20. Russakovsky O, Deng J, Su H, et al. Imagenet large scale visual recognition challenge. Int J Comput Vis. 2015;115:211-52.

21. Dietterich T. Overfitting and undercomputing in machine learning. ACM Comput Surv (CSUR). 1995;27:326-7.

22. Krizhevsky A, Sutskever I, Hinton GE. Imagenet classification with deep convolutional neural networks. Adv Neural Inf Proces Syst. 2012:1097-105.

\section{Publisher's Note}

Springer Nature remains neutral with regard to jurisdictional claims in published maps and institutional affiliations.

Ready to submit your research? Choose BMC and benefit from:
- fast, convenient online submission
- thorough peer review by experienced researchers in your field
- rapid publication on acceptance
- support for research data, including large and complex data types
- gold Open Access which fosters wider collaboration and increased citations
- maximum visibility for your research: over 100M website views per year
At BMC, research is always in progress.
Learn more biomedcentral.com/submissions

